\title{
Nonequilibrium Potential Function of Chemically Driven Single Macromolecules via Jarzynski-Type Log-Mean-Exponential Work
}

\author{
Hong Qian \\ Departments of Applied Mathematics \\ University of Washington, Seattle, WA 98195
}

October 29, 2018

\begin{abstract}
Applying the method from recently developed fluctuation theorems to the stochastic dynamics of single macromolecules in ambient fluid at constant temperature, we establish two Jarzynski-type equalities: (1) between the log-mean-exponential (LME) of the irreversible heat dissiption of a driven molecule in nonequilibrium steady-state (NESS) and $\ln P^{n e s s}(x)$, and (2) between the LME of the work done by the internal force of the molecule and nonequilibrium chemical potential function $\mu^{\text {ness }}(x)$ $\equiv U(x)+k_{B} T \ln P^{n e s s}(x)$, where $P^{n e s s}(x)$ is the NESS probability density in the phase space of the macromolecule and $U(x)$ is its internal potential function. $\Psi=$ $\int \mu^{n e s s}(x) P^{n e s s}(x) d x$ is shown to be a nonequilibrium generalization of the Helmholtz free energy and $\Delta \Psi=\Delta U-T \Delta S$ for nonequilibrium processes, where $S=-k_{B} \int P(x) \ln P(x) d x$ is the Gibbs entropy associated with $P(x)$. LME of heat dissipation generalizes the concept of entropy, and the equalities define thermodynamic potential functions for open systems far from equilibrium.
\end{abstract}

Keywords: chemical potential, fluctuation theorem, heat dissipation, nonequilibrium steady-state, stochastic macromolecular mechanics 


\section{Introduction}

One of the important concepts in equilibrium thermodynamics is chemical potential. In an equilibrium, the chemical potentials of all participating chemical components are equal. Chemical potential also plays an important role in isothermal, driven, single molecular motor systems in nonequilibrium steady-state (NESS) [1]. Potential functions for stochastic systems has been investigated previous by Graham and Haken [2, 3, 4] and recently by Ao and coworkers [5, 6]. In all the above works, the logarithm of the probability distribution in a NESS that is analogous to Boltzmann's entropy has been identified as a nonequilibrium potential function (NePf).

In classical mechanics and field theory, introducing a potential function is always associated with integration of a conservative force field. Thus, for the NePfs introduced above, one necessarily seeks an "integral interpretation". This is the objective of the present work which shows that, in a statistical sense, the integration is provided by the recent developed fluctuation theorems (FTs) and Jarzynski-type equalities in NESS [7], 8]. FTs were first developed in the context of microcanonical chaotic dynamical systems [9, 10]. It has also been studied in parallel for stochastic canonical dynamics [11, 12]. In this paper, we first present our results via a simple example: a 3-state, driven cyclic reaction widely found in biochemistry. Then the more general derivations based on the polymer representation of a macromolecule are given. Useful mathematics can be found in the Appendices.

\section{NePf and log-mean-exponential of heat and work: an example}

Fig. 1 shows a driven cyclic reaction of a single enzyme in aqueous solution at constant temperature $T$, sustained by constant concentrations of cofactors $D$ and $E$. This can be rigorously accomplished in a test tube with a feedback regenerating system, or approximately with a large excess of the cofactors [13]. In a living cell, such NESS reactions, e.g., kinase catalyzed phosphorylations and GAP-activated GTP hydrolysis [14], are ultimately maintained by the working of mitochondria which constantly regenerate ATP from ADP.

The chemical potential for a species $X(=D$ or $E)$ is $\mu_{X}=\mu_{X}^{o}+k_{B} T \ln c_{X}$ where $c_{X}$ is the 
concentration for the species $X$. Then it is easy to show that

$$
\Delta \mu_{E D}=\mu_{D}-\mu_{E}=k_{B} T \ln \frac{k_{1} k_{2}^{o} k_{3} c_{D}}{k_{-1} k_{-2}^{o} k_{-3} c_{E}} .
$$

The chemical potentials for the single macromolecule are $\mu_{X}=\mu_{X}^{o}+k_{B} T \ln p_{X}$ where $p_{X}$ is the probability of the macromolecule being in state $X(=A, B$, or $C)$. If the reaction is in a closed vessel, it eventually reaches an equilibrium and $\Delta \mu_{E D}=0 \Rightarrow c_{E}^{e q} / c_{D}^{e q}=\frac{k_{-1} k_{-2}^{o} k_{-3}}{k_{1} k_{2}^{o} k_{3}}=e^{-\Delta \mu_{E D}^{0} / k_{B} T}$.

However, if one controls the reaction such that both concentrations of $D$ and $E$ are maintained at some constant values, say $c_{D}$ and $c_{E}$, then Eq. 1 gives the amount of work done to the system in order to sustain the NESS. The NESS flux

$$
\begin{aligned}
J & =k_{1} p_{A}^{\text {ness }}-k_{-1} p_{B}^{\text {ness }}=k_{2} p_{B}^{\text {ness }}-k_{-2} p_{C}^{\text {ness }}=k_{3} p_{C}^{\text {ness }}-k_{-3} p_{A}^{\text {ness }} \\
& =\frac{k_{1} k_{2}^{o} k_{3} c_{D}-k_{-1} k_{-2}^{o} k_{-3} c_{E}}{k_{1} k_{2}+k_{1} k_{3}+k_{2} k_{3}+k_{1} k_{-2}+k_{2} k_{-3}+k_{3} k_{1}+k_{-1} k_{-2}+k_{-1} k_{-3}+k_{-2} k_{-3}} .
\end{aligned}
$$

We thus have $J \times \Delta \mu_{E D}$, the work done by the external agent per unit time, $\geq 0$ and being zero if and only if when $\Delta \mu_{E D}=J=0$. The analogy between this and power $=$ current $\times$ voltage, from electrical circuits, should be noted. The work also is equal to the sum of the dissipated heat from each every isothermal reaction: $\Delta \mu_{E D}=q_{1}+q_{2}+q_{3}$ (see Fig. 1). These results reflect both the First and Second Laws of Thermodynamics in a single macromolecule far-from-equilibrium [16]. By far from equilibrium, we mean in general for a reversible reaction $X \underset{k_{-}}{\stackrel{k_{+}}{\rightleftharpoons}} Y$ in a NESS network, $J=k_{+} c_{X}-k_{-} c_{Y}=J_{+}-J_{-}$and $\Delta \mu_{Y X}=k_{B} T \ln \left(k_{+} c_{X} / k_{-} c_{Y}\right)=k_{B} T \ln \left(J_{+} / J_{-}\right)$are not linearly related [17]. However, if $J \ll J_{+}, J_{-}$, then one has $\Delta \mu \propto J$.

So far, all the materials are from standard chemical thermodynamics. (Though seldomly it has been presented in this way.) To introduce the new results from the recent work on FTs [11, 12, 15], we first define the irreversible heat dissipation following a stochastic trajectory in a NESS, $Q_{i r r}(t)$. Let the single macromolecule having the trajectory $x_{0}, x_{1}, \ldots, x_{n}\left(x_{i}=A, B\right.$, or $\left.C\right)$ with transition from state $x_{i-1}$ to state $x_{i}$ at $t_{i}\left(t_{0}=0<t_{1}<\ldots<t_{n}<t\right)$, and defining

$$
Q_{i r r}(t)=-\sum_{i=1}^{n} \Delta \mu_{x_{i-1} x_{i}}
$$


where $\Delta \mu_{A B}=q_{1}, \Delta \mu_{B C}=q_{2}$, and $\Delta \mu_{C A}=q_{3}$, all positive. $Q_{i r r}(t)$ is called irreversible heat since the dissipation here are truly irreversible: $Q_{i r r}(t) \equiv 0$ in an equilibrium steady-state. There is, however, a fluctuating reversible heat exchange between the system and its environment even in equilibrium. The latter term is not contained in the $Q_{i r r}(t)$. In a NESS, for each every reaction $\Delta \mu$ $=\Delta h^{o}-T \Delta s^{o}-T \Delta s_{\text {concentration }}$, hence the total heat dissipation $=\Delta \mu-\Delta h^{o}=-T \Delta s$, where entropy change $\Delta s$ includes macromolecular structural entropy change $\Delta s^{o}$ and concentration

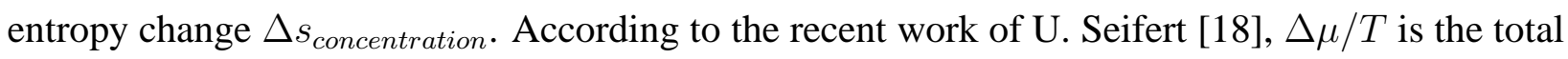
(irreversible) entropy production, $\Delta s$ is the entropy change, and $\Delta h^{o} / T$ is the exchanged heat which can be positive or negative. If the reaction is in an isothermal equilibrium, $\Delta \mu=0$ and fluctuation in heat exchange is equal to the enthalpy fluctuation along a stochastic trajectory. This exact compensation between fluctuating entropy and enthalpy was first discussed in [19]. It is a direct consequence of microscopic energy conservation.

Applying the methods developed for FTs [15, 18, 20, 21], it can be shown that

$$
\ln p_{X}^{\text {ness }}=\ln \left\langle\delta_{X, x_{n}} e^{-Q_{i r r}(t) / k_{B} T}\right\rangle
$$

where $\langle\cdots\rangle$ is ensemble average over all the possible trajectories with initial distribution $p^{\text {ness }}$ for $x_{0}$, and $\delta_{i, j}$ is the Kronecker-delta function for discrete $i$ and $j$. This is a new result. In the light of Jarzynski's equality and the work of Hummer and Szabo [7, 22], Eq. 4] firmly establishes $\ln p^{\text {ness }}$ as a NePf of the NESS. More specifically, these previous studies have shown that the integration of work (or heat), though itself is not path-independent and a function of the state, its LME is a function of state. Jarzynski in fact has shown that the LME of work is the free energy of the system. Now Eq. 4 shows that the LME of heat dissipation is a generalization of Boltzmann's entropy. Note that for equilibrium steady-state, $Q_{i r r}(t) \equiv 0$ and Eq. 4 becomes a trivial equality.

As the celebrated Jarzynski's equality, Eq. 4 is valid for all finite $t$. We note that Eq. 4 does not provide a novel way to compute $p^{\text {ness }}$ since computing heat dissipations, the $q$ 's, requires knowing $p^{\text {ness }}$ a priori (Fig. 1). However, if we replace $Q_{i r r}(t)$ by the internal work $W_{\text {int }}(t)=$ $-\sum_{i=1}^{n} \Delta \mu_{x_{i-1} x_{i}}^{o}=-\mu_{x_{n}}^{o}+\mu_{x_{0}}^{o}$, then we have

$$
k_{B} T \ln \left\langle\delta_{X, x_{n}} e^{-W_{i n t}(t) / k_{B} T}\right\rangle^{x_{0}}=\left(\mu_{X}^{o}+k_{B} T \ln p_{X}(t)\right)-\left(\mu_{x_{0}}^{o}+k_{B} T \ln p_{x_{0}}(0)\right),
$$

where $\langle\cdots\rangle^{x_{0}}$ denotes ensemble average over all possible trajectories which start at $x_{0}$. The right- 
hand-side is the chemical potential difference between $X$ at time $t$ and $x_{0}$ at time zero. This result resemble very much the Jarzynski equality, in which the two end states are equilibrium states, while here the two end states are two configurations of a NESS.

\section{NePf based on stochastic macromolecule mechanics of driven single molecules}

The above results for the 3-state driven system can be generalized to any single macromolecules in aqueous solution with stochastic dynamics, either in terms of discrete master equations [23] or continuous Brownian dynamics [24, 19]. The discrete approach is a tradition of chemical kinetics. For a single macromolecule, the kinetics consists of unimolecular reactions with first-order rate equations and pseudo-first-order rate equations when cofactors are involved. The continuous Brownian (Langevin) approach to the dynamics of single macromolecules began with Kramer's theory [25] and has been extensively studied in polymer dynamics [26]. We shall develop the general theory of NePf based on the Brownian dynamic formalism below.

Based on the polymer dynamic approach, we have recently developed a nonequilibrium thermodynamic theory for overdamped single macromolecules, such as a motor protein, in aqueous solution, which we called stochastic macromolecular mechanics [19, 27, 28]. We have shown how thermodynamic concepts such as entropy production rate, heat dissipation rate, and Onsager's thermodynamic force are rigorously developed from the dynamic equation [19]. Furthermore, we have introduced mesoscopic, trajectory-based fluctuating energy $U_{t} \equiv U\left(X_{t}\right)$ and entropy $\Upsilon_{t} \equiv-k_{B} \ln P\left(X_{t}\right)$ where $X_{t}$ is a stochastic trajectory of a macromolecule in its $3 N$-dimensional phase space and $P(x)$ is the probability density. We observed that in an isothermal equilibrium $U_{t}-T \Upsilon_{t} \equiv 0$ along a stochastic trajectory (Recall the above example.) Beyond equilibrium steady-state, we have suggested that $\mu(x) \equiv U(x)-T \Upsilon(x)$ as the nonequilibrium chemical potential function for the macromolecule in its driven NESS. Introducing the fluctuating entropy $\Upsilon_{t}$ in terms of the $P(x)$, however, is not completely satisfying since it requires the probability density being solved from a Fokker-Planck equation a priori. Also from an experimental standpoint as well as a molecular simulation standpoint, this definition is not very useful since it can not be obtained along stochastic trajectories $X_{t}$. The fluctuating energy in Eq. 5] however, is computable from a stochastic trajectory (The statistical accuracy, of course, is an important but separated issue.) 
In this section we establish the NePf, $-\ln P(x)$, and the nonequilibrium chemical potential, $\mu(x)$, through FTs-like equalities (Eqs. 4 and 5] in terms of stochastic dynamics of a single macromolecule in aqueous solution [19]

$$
\eta d X_{t}=\mathbf{F}\left(X_{t}\right) d t+\sqrt{2 k_{B} T \eta} d B_{t}
$$

In Eq. $6 \mathbf{F}(x)$ is decomposed into an internal potential part and an external driving part: $\mathbf{F}(x)$ $=-\nabla U(x)+\mathbf{G}(x) . \eta$ is a frictional coefficient and we assume it is a scalar for simplicity. $B_{t}$ is the standard Brownian motion which represents the collision between the atoms in the macromolecule and the solvent [19, 26]. The probability density function for $X_{t}$ is the solution to a Fokker-Planck equation

$$
\partial P(x, t) / \partial t=-\nabla \cdot \mathbf{J}(x, t)=\left(k_{B} T / \eta\right) \nabla^{2} P-(1 / \eta) \nabla \cdot(\mathbf{F} P) .
$$

All FTs focus on the work done by various stochastic forces. There are three forces in our problem: the total force $\mathbf{F}$, the external force $\mathbf{G}$, and the Onsager's thermodynamic force $\Pi=$ $\mathbf{F}-k_{B} T \nabla \ln P(x, t)[19]:$

$$
W_{t o t}(t)=\int_{0}^{t} \mathbf{F}\left(X_{s}\right) \circ d X_{s}, \quad W_{\text {ext }}(t)=\int_{0}^{t} \mathbf{G}\left(X_{s}\right) \circ d X_{s}, \quad Q_{i r r}(t)=\int_{0}^{t} \boldsymbol{\Pi}\left(X_{s}, s\right) \circ d X_{s} .
$$

These stochastic integrals are called total work, external work, and irreversible heat dissipation/total entropy production, respectively. We use ‘ $\circ$ ' for Stratonovich and ‘' for Ito integrations.

If the macromolecule is in a closed vessel, i.e., $\mathbf{G}(x)=0$, then both $\mathbf{F}$ and $\Pi$ are conservative forces with potential functions $U(x)$ and $U(x)-k_{B} T \ln P(x, t)+$ Const., the internal energy and Helmholtz free energy (generalized), respectively [29]. More importantly, in equilibrium, i.e., the stationary state of the closed system, $P^{e q}(x) \propto e^{-U(x) / k_{B} T}$, and $\Pi=0$ as expected: The Onsager's thermodynamic force is zero. Then $W_{t o t}(t)$ is the fluctuating potential energy and $Q_{i r r}(t)$ is the fluctuating free energy. $Q_{i r r}(t) \equiv 0$ when the macromolecule is in an equilibrium [19].

It is easy to show that

$$
\left\langle Q_{i r r}\right\rangle(t)=\int_{0}^{t} d s \int d x \boldsymbol{\Pi}(x) \cdot \mathbf{J}(x, s)=\eta \int_{0}^{t} d s \int d x P^{-1} \mathbf{J}^{2}(x, s),
$$

is the mean entropy production which is always non-negative.

$$
\left\langle W_{t o t}\right\rangle(t)=\int_{0}^{t} d s \int d x \mathbf{F}(x) \cdot \mathbf{J}(x, s)
$$


however, is the mean heat dissipation which can be negative in transient relaxation. $W_{\text {tot }}(t)$ being the total heat dissipation has been discussed by several authors [30, 17, 19]. Finally, since

$$
W_{\text {ext }}(t)=W_{t o t}(t)-W_{\text {int }}(t)=W_{t o t}(t)+U\left(X_{t}\right)-U\left(X_{0}\right)
$$

we see that the work done by the external force is equal to the heat dissipation plus the increase in internal potential energy. This is the trajectory-based energy conservation. In a NESS the internal potential energy fluctuations and entropy fluctuations are both stationary, i.e., $E\left[U\left(X_{t}\right)\right]$ and $E\left[\ln P\left(X_{t}\right)\right]$ are constants, hence $\left\langle W_{\text {ext }}\right\rangle(t)=\left\langle W_{\text {tot }}\right\rangle(t)=\left\langle Q_{\text {irr }}\right\rangle(t)$. The work done by the external force is completely transformed into dissipated heat, which precisely equals to the isothermal entropy production, in the NESS [31].

In terms of irreversible heat $Q_{\text {irr }}(t)$, we now introduce

$$
R(x, t)=\left\langle\delta\left(x-X_{t}\right) e^{-Q_{i r r}(t) / k_{B} T}\right\rangle
$$

where $\langle\cdots\rangle$ denotes ensemble average over all possible $X_{t}$ starts with $R(x, 0)=P^{n e s s}(x)$. It then can be shown mathematically that the following equality holds true for all time $t$ (see Appendix A):

$$
\ln P^{n e s s}(x)=\ln \left\langle\delta\left(x-X_{t}\right) e^{-Q_{i r r}(t) / k_{B} T}\right\rangle,
$$

which is our main result. It proves Eq. 4. The trajectory-based log-mean-exponential (LME) of the irreversible heat dissipation is the NePf , as previously suggested by Graham [4], Ao [5], and Qian [1]. Note that the "Boltzmann entropy" in Eq. 13 is defined through multiple trajectories with initial distribution $P^{n e s s}$. Alternatively with arbitrary initial distribution, Eq. 13] holds only for $t \rightarrow \infty$.

We now consider $W_{\text {int }}(t)$, the work done by the internal potential force $-\nabla U . W_{i n t}$ is related to $W_{\text {tot }}$ and and $W_{\text {ext }}(\mathrm{Eq}$. 8) and its ensemble average is zero in a NESS. The exponential average

$$
Q(x, t \mid y)=\left\langle\delta\left(x-X_{t}\right) e^{-\lambda W_{i n t}(t) / k_{B} T}\right\rangle^{y}
$$

where $\langle\cdots\rangle^{y}$ denotes ensemble average over all $X_{t}$ which start at $X_{0}=y$. Noting that $W_{\text {int }}(t)=$ $U\left(X_{0}\right)-U\left(X_{t}\right)$, it is easy to see that

$$
k_{B} T \ln Q(x, t \mid y)=U(x)+k_{B} T \ln P(x, t \mid y)-U(y) \equiv \mu(x, t)+\text { const. }
$$


is, off by a constant, the nonequilibrium chemical potential at time $t$. In other words, the trajectorybased LME of the internal potential energy is the nonequilibrium chemical potential. When $t \rightarrow$ $\infty$, we have

$$
k_{B} T \ln \left\langle\delta\left(x-X_{t}\right) e^{-W_{i n t}(t) / k_{B} T}\right\rangle^{y} \rightarrow \mu^{n e s s}(x) \equiv U(x)+k_{B} T \ln P^{n e s s}(x)-U(y) .
$$

Eqs. 15 and 16 for transients and the NESS of open driven systems, our second main result, are parallel to Jarzynski's equalities [7, 22] for time-dependent but detail-balanced systems.

The mathematics behind Eq. 15 is trivial since the $W_{\text {int }}$ is the work done by the internal potential force. In fact, to obtain $P(x, t)$, the simplest way is by mathematical definition $P(x, t \mid y)$ $=\left\langle\delta\left(x-X_{t}\right)\right\rangle^{y}$. We emphasize that the physically novel result is Eq.13, which defines the $\ln P^{n e s s}$ as a thermodynamic potential. The physical meaning of Eqs. 15] and 16, then, is to establish equalities between other thermodynamic potentials and the LME of other types of work in an open system. In this spirit, we have a third relation of such (Appendix B):

$$
k_{B} T \ln \left\langle\delta\left(x-X_{t}\right) e^{-W_{e x t}(t) / k_{B} T}\right\rangle^{y}=\mu(y, t)+\text { const. }
$$

where the constant is independent of $y$ and $t$; It is a function of $x$.

\section{NESS thermodynamics of single driven macromolecules: a theory}

With the chemical potential $\mu(x, t)$ defined, the free energy of a single macromolecule $\Psi(t) \equiv$ $\int \mu(x, t) P(x, t) d x$ has the fundamental property expected from a nonequilibrium generalization of Helmholtz free energy [19]:

$$
\begin{aligned}
\frac{d}{d t} \Psi(t) & =\int \nabla \mu \cdot \mathbf{J} d x=E\left[W_{e x t}(t)\right]-e_{p} \\
& =E\left[W_{\text {ext }}(t)\right]-h_{d}-T \frac{d}{d t} S
\end{aligned}
$$

where $e_{p}=\left\langle Q_{i r r}(t)\right\rangle / t=\eta \int\|\mathbf{J}\|^{2} P^{-1} d x$ is total entropy production rate, $h_{d}=\left\langle W_{t o t}(t)\right\rangle / t=$ $\int \mathbf{F} \cdot \mathbf{J} d x$ is the heat dissipation rate [19], and $S=-k_{B} T \int P \ln P d x$ is entropy. The first term in (18) is the work done by external work - energy input. The sum of the first two terms is the energy stored in the system - potential energy increase, $\Delta U$. Hence, the relation $\Delta \Psi=\Delta U-T \Delta S$ hold true even for transient driven nonequilibrium processes. In a NESS, both $d \Psi / d t$ and $d S / d t$ are 
zero and the energy input are transformed entirely into dissipated heat. Conversely, $\mu^{\text {ness }}(x)$ can be written in terms of functional differentiation, as in classic thermodynamics:

$$
\mu^{n e s s}(x)=\frac{\delta \Psi}{\delta P^{n e s s}(x)} .
$$

$\mu(x, t)$ also plays an important role in the transient dynamic relaxation to a NESS. One can introduce chemical potential difference $\mu(x, t)-\mu^{n e s s}(x)$ and its average

$$
H(t)=\int P(x, t)\left(\mu(x, t)-\mu^{n e s s}(x)\right) d x=\int P(x, t) \ln \left(\frac{P(x, t)}{P^{n e s s}(x)}\right) d x .
$$

This is a Lyapunov function for the stochastic dynamics (Eq. 7): $H(t) \geq 0$ and $(d / d t) H(t) \leq 0$ [24, 29]. Therefore, a transient process relaxing to a NESS minimizes, on average, the chemical potential difference with respect to its NESS.

Hatano and Sasa [8] have recently developed a theory for steady-state thermodynamics of stochastic systems. The relation between their work and ours [17, 19] is as follows. The key thermodynamic quantities in their theory are total heat dissipation $W_{\text {tot }}(t)$, housekeeping heat dissipation, which is precisely our $Q_{i r r}(t)$ in Eq. 8, and mesoscopic entropy $\Upsilon_{t}=-k_{B} \ln P^{n e s s}(x)$. As we have shown, the mean housekeeping heat $\left\langle Q_{i r r}\right\rangle$ is exactly the entropy production rate $\left(e_{p}\right)$ in our work [17, 19], this relation is further explored in [32]. Their irreversible force $b(x)$ $=G(x)-\nabla \mu^{\text {ness }}(x)$ is exactly $\Pi$, the Onsager's thermodynamic force. We derived an entropy balance equation in a NESS $d S / d t=e_{p}-E\left[W_{t o t}(t)\right]$ which they called excess heat $Q_{e x}$. Their study, however, has gone beyond simple NESS systems and their main result is concerned with time-dependent driven system, i.e, $\mathbf{F}$ in (6) is also an explicit function of $t$. In parallel with [7] and treating $\Upsilon(x, t)$ as a time-dependent potential energy function, they introduced a charging process

$$
Y(t)=\int_{0}^{t} \frac{\partial \Upsilon}{\partial s}\left(X_{s}, s\right) d s=\frac{1}{T} \int_{0}^{t} \frac{\partial\left(U-\mu^{n e s s}\right)}{\partial s}\left(X_{s}, s\right) d s
$$

and found an interesting generalization of Jarzynski's result to driven systems. The mean $\langle Y(t)\rangle$ is a generalization of the relative entropy $H(t)$ in (20). The present work, however, is limited to time-independent $\mathbf{F}(x)$ and is concerned with establishing the concept of NePf and nonequilibrium chemical potential in terms of the LME of certain type of work in a nonequilibrium open system.

\section{Discussions}


Stochastic NESS circuit theory. An analogy between the nonequilibrium steady-state thermodynamics and electrical network theory exists. $\mu$ represents potentials, $\nabla \mu$ represents potential gradient, $\mathbf{J}$ represents flux, and $\nabla \cdot \mathbf{J}=0$ is Kirchhoff's current law. Tellegen's theorem then says that $\nabla \mu$, is perpendicular to $\mathbf{J}$ in a NESS:

$$
\int \nabla \mu(x) \cdot \mathbf{J}(x) d x=-\int \mu(x) \nabla \cdot \mathbf{J}(x) d x=0 .
$$

In the case of a system being driven at its boundary, $\mathbf{F}(x)=-\nabla U(x)$ for $x \in \Omega$ and the nonequilibrium driving force is only imposed on the boundary $\partial \Omega$ :

$$
\int_{\Omega} \nabla \mu(x) \cdot \mathbf{J}(x) d x=\int_{\partial \Omega} \mu(x) \mathbf{J}(x) \cdot d \mathbf{S}
$$

is analogous to Kirchhoff's potential law. For $x \in \partial \Omega, \mathbf{J} \neq 0$ is called flux injection and $\mu(x) \neq 0$ is called potential clamping [31, 33, 34]. If there is neither flux injection nor potential clamping, then the system $(x \in \Omega)$ is closed and it reaches equilibrium with $\mathbf{J}=\nabla \mu=0$.

Graham-Haken decomposition. In the present work, the decomposition of nonconservative force $\mathbf{F}(x)=-\nabla U(x)+\mathbf{G}(x)$ is based on the physical reality of open and closed systems: Any open system has a unique corresponding closed system with internal potential $U(x)$. Knowing this correspondence greatly enhances the thermodynamic understanding of the open system (see [1, 15]). Mathematically, however, Helmholtz-Hodge decomposition $\mathbf{F}=-\nabla \phi+\gamma$ is not unique. Graham and Haken [2] have shown that if one chooses $\phi=-k_{B} T \ln P^{\text {ness }}(x)$, then $\gamma$ satisfies $\nabla \cdot \gamma-\nabla \phi \cdot \gamma=0$. In terms of this particular decomposition, our $\mu(x)$ in Eq. $[15] \equiv$ const., and more importantly, $Q^{g h}(x, t)=\int Q(x, t \mid y) d y \equiv 1$. That is, the exponential average of Graham-Haken "internal work", over an initially uniform distribution, is 1 for any finite time $t$. The significance of this result is that one, having experimental or simulated realizations of many trajectories of $X_{t}$ with finite time duration $X_{t}\left(0 \leq t \leq t^{*}\right)$, can devise an inverse algorithm to construct $\phi(x)$ such that

$$
k_{B} T \ln \left\langle\delta\left(x-X_{t}\right) e^{\left(\phi\left(X_{t}\right)-\phi\left(X_{0}\right)\right) / k_{B} T}\right\rangle \equiv 0 .
$$

Finally, we notice that if one chooses the Graham-Haken decomposition, then by the result in footnote 3, we have

$$
\left\langle\delta\left(y-X_{t}\right) e^{-\int_{0}^{t} \gamma\left(X_{s}\right) \circ d X_{s}}\right\rangle^{x}=\left\langle\delta\left(x-X_{t}\right) e^{\int_{0}^{t} \nabla \phi\left(X_{s}\right) \circ d X_{s}}\right\rangle^{y} \rightarrow P^{n e s s}(y)
$$


in the limit of $t \rightarrow \infty$.

Umbrella sampling in stochastic dynamics. Umbrella sampling is an important method in molecular simulations [35]. Our result on nonequilibrium chemical potential also has an intimate relation to a more general mathematical result which has applications for umbrella sampling. Consider an arbitrary function $V(x)$ and integral $I_{V}(t)=-\int_{0}^{t} \nabla V\left(X_{s}\right) \circ d X_{s}$, then

$$
\left\langle\left\langle\delta\left(x-X_{s}\right) e^{-I_{V}(t) / k_{B} T}\right\rangle^{y} e^{(V(y)-V(x)) / k_{B} T}\right\rangle=P^{n e s s}(x)
$$

in which the inner $\langle\cdots\rangle^{y}$ is an averaging over all $X_{t}$ with $X_{0}=y$ and the outer $\langle\cdots\rangle$ is an averaging over all $X_{0}$ with the stationary distribution of $X$. The result is the stationary distribution which can be obtained from sampling of $X_{t}$ weighted toward those trajectories which move along with the gradient of $V(x)$.

Conclusions. Open, driven chemical and molecular systems far from chemical equilibrium have been studied from several different points of view in statistical physics. In terms of stochastic processes, these investigations include "nonequilibrium potentials" [1, 4, 5], "dissipative structures" [36], "cycle kinetics" [16, 37, 38], "Brownian motors" [39, 16], "stochastic resonance" [40], and "fluctuation theorems"[11, 12, 15, 18]. The result we obtain in this work, in terms of a Jarzynski-type equality, clearly demonstrates an intimate connection between the fluctuation theorems and the nonequilibrium potentials. Combining with previous work which has shown relations between Brownian motors and cycle kinetics [41], between cycle kinetics and fluctuation theorems [17], between Brownian motors and excess entropy [42], and between stochastic resonance and cycle kinetics [43], a consistent and comprehensive statistical thermodynamic theory for single macromolecules far from equilibrium emerges. Finally but not least, a variational principle for NESS in terms of LME of heat dissipation seems to be in sight.

I thank P. Ao, Z.Q. Chen, R. Fox, G. Gallavotti, R. Graham, C. Jarzynski, S.-i. Sasa, J.M. Schurr, U. Seifert, Z.F. Shao, and X.S. Xie for helpful discussions.

\section{References}

[1] Qian, H. Phys. Rev. E. 2004, 69, 012901. 
[2] Graham, R., Haken, H. Zeit. Phys. A. 1971, 243, 289-302.

[3] Graham, R., Tél, T. Phys. Rev. A. 1985, 31, 1109-1122.

[4] Graham, R. In Noise in Nonlinear Dynamical Systems; Moss, F., McClintock, P.V.E., Eds.; Cambridge Univ. Press: London, 1989, pp. 225-278.

[5] Ao, P. J. Phys. A. 2004, 37, L25-L30.

[6] Kwon, C.-L., Ao, P., Thouless, D.J. Proc. Natl. Acad. Sci. U.S.A. 2005, 192, 13029-13033.

[7] Jarzynski, C. Phys. Rev. Lett. 1997, 78, 2690-2693.

[8] Hatano, T., Sasa, S.-i. Phys. Rev. Lett. 2001, 86, 3463-3466.

[9] Gallavotti, G., Cohen, E.G.D. Phys. Rev. Lett. 1995, 74, 2694-2697.

[10] Gallavotti, G. Phys. Rev. Lett. 1996, 77, 4334-4337.

[11] Kurchan, J. J. Phys. A. 1998, 31, 3719-3729.

[12] Lebowitz, J.L., Spohn, H. J. Stat. Phys. 1999, 95, 333-365.

[13] Xie, X.S., Lu, H.P. J. Biol. Chem. 1999, 274, 15967-15970.

[14] Qian, H. Biophys. Chem. 2003, 105, 585-593.

[15] Crooks, G. Phys. Rev. E. 1999, 60, 2721-2726.

[16] Qian, H. J. Phys. Cond. Matt. 2005, in the press.

[17] Qian, H. Phys. Rev. E. 2001, 65, 022101.

[18] Seifert, U. Phys. Rev. Lett. 2005, 95, 040602.

[19] Qian, H. Phys. Rev. E. 2001, 65, 016102.

[20] Jiang, D.-Q., Qian, M., Zhang, F.-X. J. Math. Phys. 2003, 44, 4174-4188.

[21] Gaspard, P. J. Chem. Phys. 2004, 120, 8898-8905. 
[22] Hummer, G., Szabo, A. Proc. Natl. Acad. Sci. USA 2001, 98, 3658-3661.

[23] Schnakenberg, J. Rev. Mod. Phys. 1976, 48, 571-585.

[24] Bergmann, P.G., Lebowitz, J.L. Phys. Rev. 1955, 99, 578-587.

[25] Hänggi, P., Talkner, P., Borkovec, M. Rev Mod Phys 1990, 62, 251-341.

[26] Doi, M., Edwards, S.F. The Theory of Polymer Dynamics, Clarendon: Oxford, 1986.

[27] Qian, H. J. Phys. Chem. B. 2002, 106, 2065-2073.

[28] Qian, H., Qian, M., Tang, X. J. Stat. Phys. 2002, 107, 1129-1141.

[29] Qian, H. Phys. Rev. E. 2001, 63, 042103.

[30] Sekimoto, K. J. Phys. Soc. Jpn. 1997, 66, 1234-1237.

[31] Qian, H., Beard, D.A. Biophys. Chem. 2005, 114, 213-220.

[32] Speck, T., Seifert, U. J. Phys. A. 2005, cond-mat/0507420.

[33] Qian, H., Beard, D.A., Liang, S.D. Eur. J. Biochem. 2003, 270, 415-421.

[34] Beard, D.A., Liang, S.D., Qian, H. Biophys. J. 2002, 83, 79-86.

[35] Chandler, D. Introduction to Modern Statistical Mechanics, Oxford Univ. Press: New York, 1987.

[36] Nicolis, G., Prigogine, I. Self-organization in Nonequilibrium Systems, John-Wiley \& Sons: New York, 1977.

[37] Hill, T.L. Free Energy Transduction in Biology, Academic Press: New York, 1977.

[38] Hill, T.L. Free Energy Transduction and Biochemical Cycle Kinetics, Springer-Verlag: New York, 1989.

[39] Jülicher, F., Ajdari, A., Prost, J. Rev. Mod. Phys. 1997, 69, 1269-1281. 
[40] Gammaitoni, L., Hänggi, P., Jung, P., Marchesoni, F. Rev. Mod. Phys. 1998, 70, 223-287.

[41] H. Qian, Phys. Rev. Lett. 1998, 81, 3063-3066.

[42] H. Qian, Phys. Rev. E. 2002, 65, 021111.

[43] H. Qian and M. Qian, Phys. Rev. Lett. 2000, 84, 2271-2274.

[44] Øksendal, B. Stochastic Differential Equations, 4th Ed. Springer: New York, 1995.

\section{Appendices}

In stochastic mathematics, Langevin equations such as in Eq. 6 are known as stochastic differential equations [44]. Because the random force $d B_{t}$, a solution to the equation is stochastic and has to be characterized in terms of a probability density function $P(x, t)$ which satisfies the corresponding Eq. 7. For arbitrary force $\mathbf{K}(x, t)$, let $\phi(x, t)=\left\langle\delta\left(x-X_{t}\right) e^{-\int_{0}^{t} \mathbf{K}\left(X_{t}, t\right) \circ d X_{t} / k_{B} T}\right\rangle$ where $\langle\cdots\rangle$ denotes ensemble average and let $\widehat{\phi}(t, x)=\left\langle e^{-\int_{0}^{t} \mathbf{K}\left(X_{t}, t\right) \circ d X_{t} / k_{B} T}\right\rangle^{x}$ where $\langle\cdots\rangle^{x}$ denotes ensemble average over all possible trajectories (solutions to Eq. 6) which start at $x$, the CameronMartin-Girsanov formula [44] states that $\widehat{\phi}(t, x)$ satisfies the equation $\partial \widehat{\phi} / \partial t=\mathcal{L}^{*} \widehat{\phi}$ and $\phi(x, t)$ satisfies the equation $\partial \phi / \partial t=\mathcal{L} \phi$, where the elliptic operator $\mathcal{L}=\left(k_{B} T / \eta\right) \nabla^{2}-(1 / \eta)[\mathbf{F} \cdot \nabla+$ $\left.\nabla \cdot \mathbf{F}-2 \mathbf{K} \cdot \nabla-\nabla \cdot \mathbf{K}-\left(1 / k_{B} T\right) \mathbf{K} \cdot(\mathbf{K}-\mathbf{F})\right]$, and $\mathcal{L}^{*}$ is the adjoint of $\mathcal{L}$.

Now for arbitrary scale $q(x, t)$, let $\psi(x, t)=\left\langle\delta\left(x-X_{t}\right) e^{-\int_{0}^{t} q\left(X_{s}, s\right) d s / k_{B} T}\right\rangle$ and $\widehat{\psi}(t, x)=$ $\left\langle e^{-\int_{0}^{t} q\left(X_{s}, s\right) d s / k_{B} T}\right\rangle^{x}$, the Feymann-Kac formula [44] states that $\widehat{\psi}(t, x)$ satisfies the equation $\partial \widehat{\psi} / \partial t$ $=\mathcal{L}^{*} \widehat{\psi}$ and $\psi(x, t)$ satisfies the equation $\partial \psi / \partial t=\mathcal{L} \psi$, where the operator $\mathcal{L}=\left(k_{B} T / \eta\right) \nabla^{2}-$ $(1 / \eta)[\mathbf{F} \cdot \nabla+\nabla \cdot \mathbf{F}]-\left(1 / k_{B} T\right) q(x, t)$. These two adjoint relations are behind the work of Jarzynski [7] and that of Hummer and Szabo [22]. In those studies, the work $W(t)$ is defined through an integral $\int_{0}^{t} F\left(X_{s}, s\right) v(s) d s$ where $F(x, s)$ is a time-dependent external force and $v(s)$ is a deterministic rate. Hence Feymann-Kac, instead of Girsanov, formula was used.

Appendix A. $R(x, t)=\left\langle\delta\left(x-X_{t}\right) e^{-Q_{i r r}(t) / k_{B} T}\right\rangle$ with $R(x, 0)=P^{n e s s}(x)$. That is the above $\mathbf{K}=\boldsymbol{\Pi}$ in Eq. 8. Hence $R(x, t)$ satisfies the partial differential equation $\partial R(x, t) / \partial t=\mathcal{L} R(x, t)$ where

$$
\mathcal{L}=\frac{k_{B} T}{\eta} \nabla^{2}-\frac{1}{\eta}\left[\mathbf{F} \cdot \nabla+(\nabla \cdot \mathbf{F})-2 \boldsymbol{\Pi} \cdot\left(\nabla-\nabla \ln P^{n e s s}\right)\right]
$$


It is easy to verify that $P^{n e s s}(x)$ is the solution to this equation for all time $t$. Hence we arrive at Eq. 13 .

Appendix B. If the $\mathbf{F}$ in Eq. 6 has $\mathbf{F}=\mathbf{F}_{1}+\mathbf{F}_{2}$, then applying the above result we have $\left\langle\delta\left(x-X_{t}\right) e^{-\int_{0}^{t} \mathbf{F}_{1}\left(X_{s}\right) \circ d X_{s}}\right\rangle^{y}=\left\langle\delta\left(y-X_{t}\right) e^{-\int_{0}^{t} \mathbf{F}_{2}\left(X_{s}\right) \circ d X_{s}}\right\rangle^{x}$. A direct consequence of this result is a symmetry relation $\left\langle\delta\left(x-X_{t}\right) e^{-\lambda W_{t o t}(t)}\right\rangle^{y}=\left\langle\delta\left(y-X_{t}\right) e^{-(1-\lambda) W_{t o t}(t)}\right\rangle^{x}$. This is an alternative statement of the FTs.

Now let $Z(y, t)=\left\langle\delta\left(x-X_{t}\right) e^{-W_{e x t}(t) / k_{B} T}\right\rangle^{y}$. As a function of $y$, it satisfies the Kolmogorov backward equation $\partial Z(y, t) / \partial t=\mathcal{M} Z(y, t)$ where the operator $\mathcal{M}=\left(k_{B} T / \eta\right) \nabla^{2}-(1 / \eta)[\mathbf{F} \cdot \nabla+$ $\left.(\nabla \cdot \mathbf{F})+2(\nabla U) \cdot \nabla+\nabla^{2} U\right]+\left(1 / \eta k_{B} T\right) \nabla U \cdot \mathbf{G}=e^{U / k_{B} T} \mathcal{L}_{0} e^{-U / k_{B} T} \cdot \mathcal{L}_{0}$ is the right-hand-side of Eq.7. Because $W_{\text {ext }}+W_{\text {int }}=W_{\text {tot }}$, we arrive at Eq. 17 


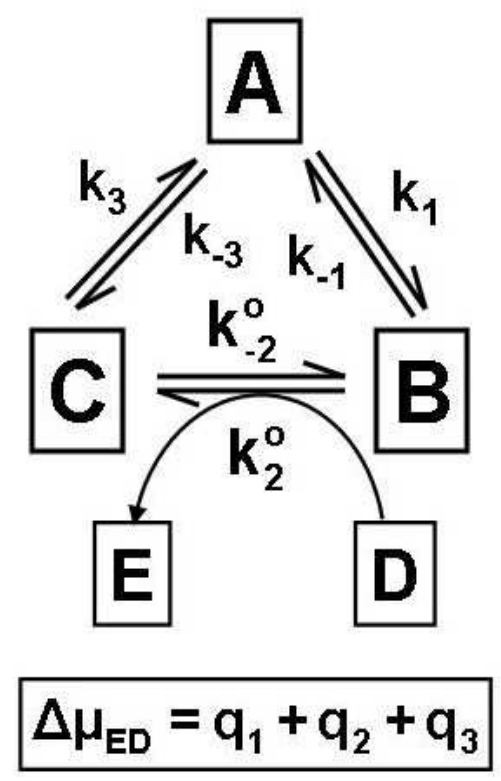

Figure 1: A single macromolecule can be in states $A, B$, and $C$. The transition from $B$ to $C$ is accompanied by the turnover of a cofactor between $D$ and $E$ via the second-order $k_{2}^{o}$ and $k_{-2}^{o}$. We denote pseudo-first-order rate constants $k_{2}^{o} c_{D}=k_{2}$ and $k_{-2}^{o} c_{E}=k_{-2}$. The total chemical driving force for the cyclic reaction is $\Delta \mu_{E D}$ given in Eq. 1. $q_{1}=k_{B} T \ln \left(p_{A} k_{1} / p_{B} k_{-1}\right), q_{2}=$ $k_{B} T \ln \left(p_{B} k_{2} / p_{C} k_{-2}\right)$, and $q_{1}=k_{B} T \ln \left(p_{C} k_{3} / p_{A} k_{-3}\right)$, are the heat dissipation associated with steps 1,2 , and 3 , respectively. The equality in the box reflects the conservation of energy. 